\title{
Regularized Iterative Weighted Filtered Back-Projection for Few-View Data Photoacoustic Imaging
}

\author{
Xueyan Liu ${ }^{1}$ and Dong Peng ${ }^{2}$ \\ ${ }^{1}$ Department of Mathematics Science, Liaocheng University, Liaocheng 252000, China \\ ${ }^{2}$ Life Sciences Research Center, School of Life Sciences and Technology, Xidian University, Xian 710071, China \\ Correspondence should be addressed to Xueyan Liu; liuxueyan@fingerpass.net.cn
}

Received 17 February 2016; Revised 16 June 2016; Accepted 19 June 2016

Academic Editor: Giancarlo Ferrigno

Copyright (c) 2016 X. Liu and D. Peng. This is an open access article distributed under the Creative Commons Attribution License, which permits unrestricted use, distribution, and reproduction in any medium, provided the original work is properly cited.

\begin{abstract}
Photoacoustic imaging is an emerging noninvasive imaging technique with great potential for a wide range of biomedical imaging applications. However, with few-view data the filtered back-projection method will create streak artifacts. In this study, the regularized iterative weighted filtered back-projection method was applied to our photoacoustic imaging of the optical absorption in phantom from few-view data. This method is based on iterative application of a nonexact 2DFBP. By adding a regularization operation in the iterative loop, the streak artifacts have been reduced to a great extent and the convergence properties of the iterative scheme have been improved. Results of numerical simulations demonstrated that the proposed method was superior to the iterative FBP method in terms of both accuracy and robustness to noise. The quantitative image evaluation studies have shown that the proposed method outperforms conventional iterative methods.
\end{abstract}

\section{Introduction}

Photoacoustic imaging (PAI) combining good acoustic resolution with high optical contrast in a single modality has great potential for tremendous clinical applications [1]. It is promising in many aspects, for example, the detection of breast cancer, skin cancer, and osteoarthritis in humans [24]. In the past decades, many algorithms have been proposed for image reconstruction when the ultrasonic transducer collects signals from a full view [5-7]. A limiting factor for these algorithms is a large number of measurements made with transducers. In addition, in many potential applications of PAI, such as ophthalmic imaging and breast imaging, the object is only accessible from limited angles. A practical need exists for reconstruction from few-view data, as this can greatly reduce the required scanning time and the number of ultrasound sensors [8-11].

Analytic algorithms like filtered back-projection (FBP) and time-reversal based reconstruction attain very fast reconstruction performance $[5,12]$. However, these algorithms have an inherent limitation of requiring large number of data points around the target object for accurately estimating the optical absorption. And implementations of such formulae may cause streak-type artifacts and negative values in the reconstructed image. To overcome these limitations, iterative image reconstruction algorithms have been proposed to improve the reconstructed image quality [13-16], which can mitigate artifacts from incomplete few-view data and permit reductions in data-acquisition times. And iterative methods can be significantly accelerated with GPU-based reconstructions [17]. Because of this, the development of iterative image reconstruction algorithms for PAI is an important research topic of current interest. By minimizing the least-square error between the measured signals and the signals predicted by the exact photoacoustic propagation model, the model-based photoacoustic inversion method has been proved to be stable and accurate. However, its reconstruction is computationally burdensome which limits its application in the practical PAI [16-18]. Finally, iterative weighted algorithms can effectively mitigate image artifacts due to limited-view acoustic data [1921]. All of these methods provide the opportunity for accurate image reconstruction from few-view data.

In this study, inspired by the iterative weighted approaches in CT $[20,22,23]$, we derived a regularized iterative weighted FBP (RIWFBP) method to improve the convergence properties of the iterative loop and improve 
image quality in few-view PAI. During the reconstruction, we firstly use the effective few-view scanning angle improved FBP method to reconstruct an initial image of the optical absorption [24]. In each iteration step, the difference between the collected signals and the calculated signals was used to update the correction image, and a regularization operation that improved the convergence properties of the iterative loop was added. Numerical simulation and experimental results reveal the good performance of the RIWFBP method.

This paper is organized as follows. In Section 2, the iterative improved FBP method and the regularized enhanced iterative scheme are reviewed briefly. In Section 3, besides using numerical phantoms, we also conducted experimental measurements and applied our reconstruction method to the obtained data. Finally, the conclusions are drawn in Section 4.

\section{Methods}

2.1. Iterative Improved Filtered Back-Projection Method. According to the forward problem for an acoustically homogeneous model present in [8], the acoustic wave pressure $p\left(r_{0}, t\right)$ at a detector position $r_{0}$ and time $t$ over a circle in the $2 \mathrm{D}$ imaging is related to the spatial distribution of electromagnetic absorption $A(r)$,

$$
p\left(r_{0}, t\right)=\frac{c \beta I}{4 \pi C_{p}} \frac{\partial}{\partial t} \oint_{t=\left|r-r_{0}\right| / c} \frac{A(r)}{\left|r-r_{0}\right|} d r .
$$

Here $\beta$ is the coefficient of volumetric thermal expansion; $C_{p}$ is the isobaric specific heat; $c$ is the speed of sound; and $I$ is the temporal profile. For 2D imaging, the approximate inverse solution for the circular-scan geometry can be represented by [25]

$$
A(r)=-\left.\frac{r_{0}^{2} C_{p}}{2 \pi \beta c^{4}} \int_{\varphi_{0}} d \varphi_{0} \frac{1}{t} \frac{\partial p\left(r_{0}, t\right)}{\partial t}\right|_{t=\left|r_{0}-r\right| / c}
$$

To numerically model the above forward and inverse problems, we used vector $x$ to represent $A(r)$ and vector $y$ to represent $p\left(r_{0}, t\right)$. Then the forward problem can be described as $y=P x$, and the reconstruction formula can be written as $x=R P x$ [5], where $R$ is the back-projection operator [8]. In real biological tissue imaging only the noisy signals can be detected from few-view data. $P$ is an ill-conditioned matrix; thus we cannot obtain an exact image.

Iterative improved FBP (IFBP) methods have been used to reduce artifacts due to an insufficient data and streaks due to missing angles. The update step of IFBP is then given by [13]

$$
x^{m}=x^{m-1}+\alpha R\left(y-P x^{m-1}\right), \quad m=1,2,3, \ldots .
$$

In this way, a sequence of image vectors is produced.

\subsection{Regularized Iterative Weighted Filtered Back-Projection} Method. In this section, we will present the RIWFBP method for the few-view PAI imaging. This contribution is an extension of theory and experiments on iterative weighted FBP (IWFBP) presented in [26]. By using the effective scanning angle the algorithm for full-view data can be approximately extended to the few-view case. The reconstructed intensity error problem induced by few-view scanning can be improved [24]

$$
A(r) \approx-\left.\frac{r_{0}^{2} C_{p}}{2 \pi \beta c^{4}} \int_{\theta_{1}}^{\theta_{2}} d \theta \frac{1}{\theta_{e} t} \frac{\partial p\left(r_{0}, t\right)}{\partial t}\right|_{t=\left|r_{0}-r\right| / c},
$$

where $\theta_{e}$ is the effective scanning angle and $\theta_{1}$ and $\theta_{2}$ are, respectively, the minimum and maximum angle of the signal acquisition position. Based on (4), we used the RIWFBP method to compensate for the nonexactness of FBP.

During the reconstruction, we firstly used $\theta_{e}$ weighted FBP method to reconstruct an initial distribution $x^{0}$ of the absorbed energy density. Then we applied the RIWFBP method to update the distribution $x^{m}$ of the absorbed energy density. To compensate for the difference between the reconstruction $x^{m}$ and the actual image $x$, a weighted parameter [26],

$$
\lambda_{m}=\frac{\max (y)-\min (y)}{\max \left(x^{m}\right)-\min \left(x^{m}\right)}, \quad m=0,1,2, \ldots,
$$

was used to correct the differences between the measured signals $y$ and computed signals $P x^{m}$. We obtained the error correction image $\Delta x^{m}$ from the differences between $y$ and $\lambda_{m-1} P x^{m}$ at each iteration step. The recursion expression is as follows:

$$
\begin{array}{r}
x^{m}=x^{m-1}+\Delta x^{m}=x^{m-1}+\alpha R\left(y-\lambda_{m-1} P x^{m-1}\right), \\
m=1,2, \ldots .
\end{array}
$$

In practice, it might be wise to employ only a fraction $\alpha \epsilon$ $(0,1)$ of the full values of the correction image $\Delta x^{m}$. Inspired by the idea of regularization $[22,23]$, the RIWFBP method is designed to reconstruct the absorbed energy deposition by adding a regularization operation in the iterative loop of IFBP. This is accomplished through the quadratic regularization for least-squares minimization of the following functional:

$$
F(y, x)=\frac{1}{2}\|y-P x\|^{2}+\gamma \sum_{i=1}^{N} \sum_{j=1}^{N} d_{i j}\left(x_{i}-x_{j}\right)^{2},
$$

where $\gamma$ is a parameter determining the amount of regularization and $d_{i j}$ are the inverse distances between the pixels $i$ and $j$ in a $3^{2}$ neighborhood. The last term is obviously a penalty term. The minimization of (7) can be realized by differentiating $F$ with respect to $x_{i}$ and setting each of the resulting expressions to zero, leading to the following system of equations:

$$
\begin{array}{r}
\frac{\partial F}{\partial x_{i}}=P^{T}(y-P x)+4 \gamma \sum_{i=1}^{N}\left(\sum_{j=1}^{N} d_{i j}\left(x_{i}-x_{j}\right)\right) e_{i}=0 \\
(i=1,2, \ldots, N)
\end{array}
$$

where $\sum_{i=1}^{N}\left(\sum_{j=1}^{N} d_{i j}\left(x_{i}-x_{j}\right)\right) e_{i}=K x[22]$ and $\left\{e_{1}, e_{2}, \ldots, e_{N}\right\}$ is the standard basis for $R^{N}$. By using the steepest descent method the solution in an iterative form was given as

$$
x^{m}=x^{m-1}-\alpha\left[P^{T}\left(y-P x^{m-1}\right)\right]-4 \alpha \gamma K x^{m-1} .
$$




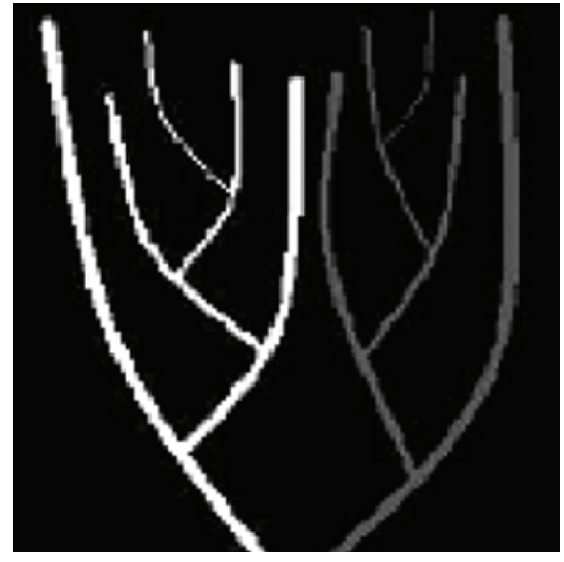

(a)

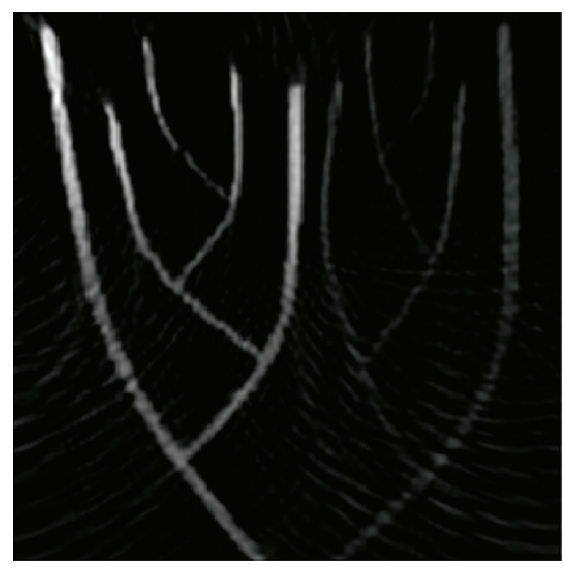

(c)

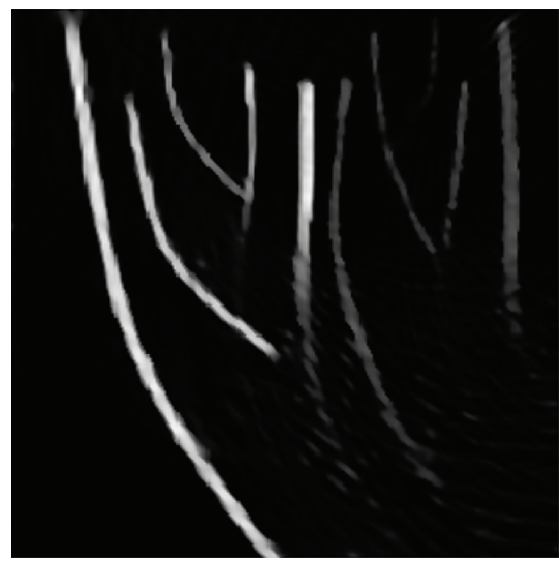

(e)
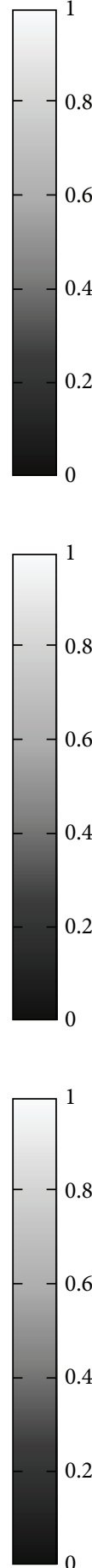

0.2

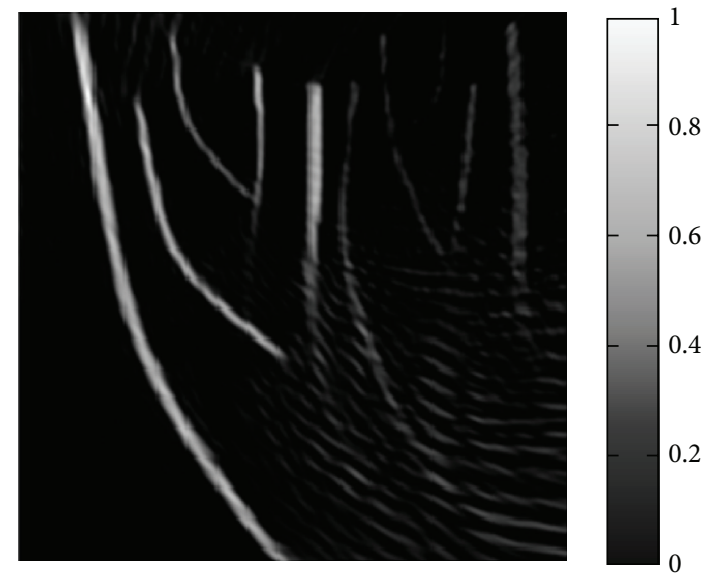

(b)

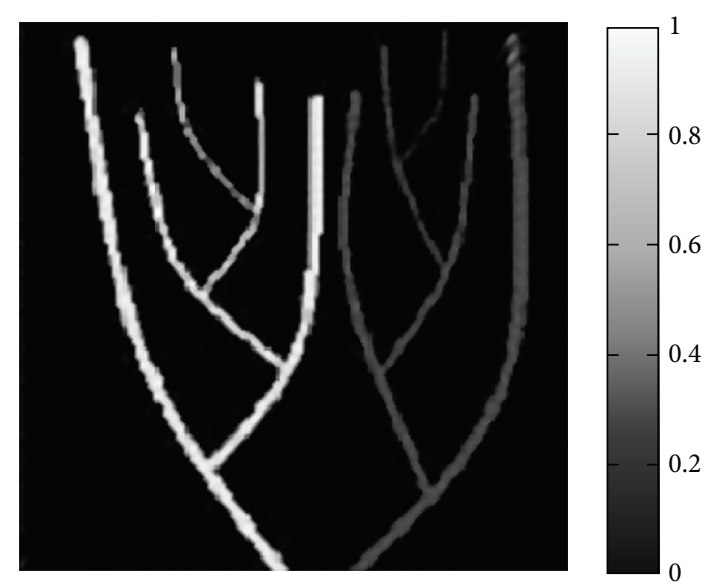

(d)
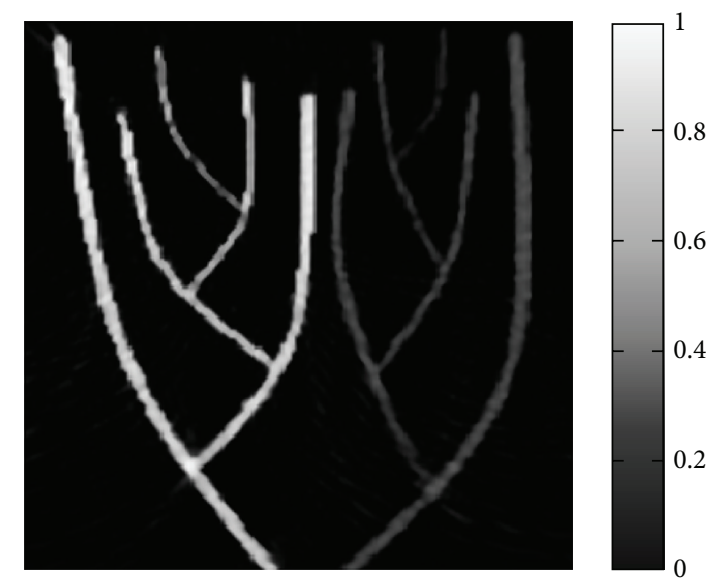

(f)

FIGURE 1: Images reconstructed from simulated data corresponding to the numerical phantom (a). (b) 30 detectors over $90^{\circ}$, using IFBP. (c) 60 detectors over $180^{\circ}$, using IFBP. (d) 120 detectors over $360^{\circ}$, using RIWFBP. (e) 30 detectors over $90^{\circ}$, using RIWFBP. (f) 60 detectors over $180^{\circ}$, using RIWFBP. 


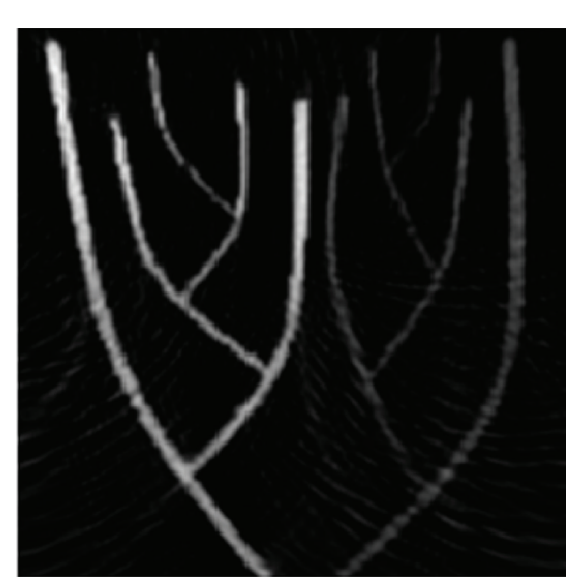

(a)
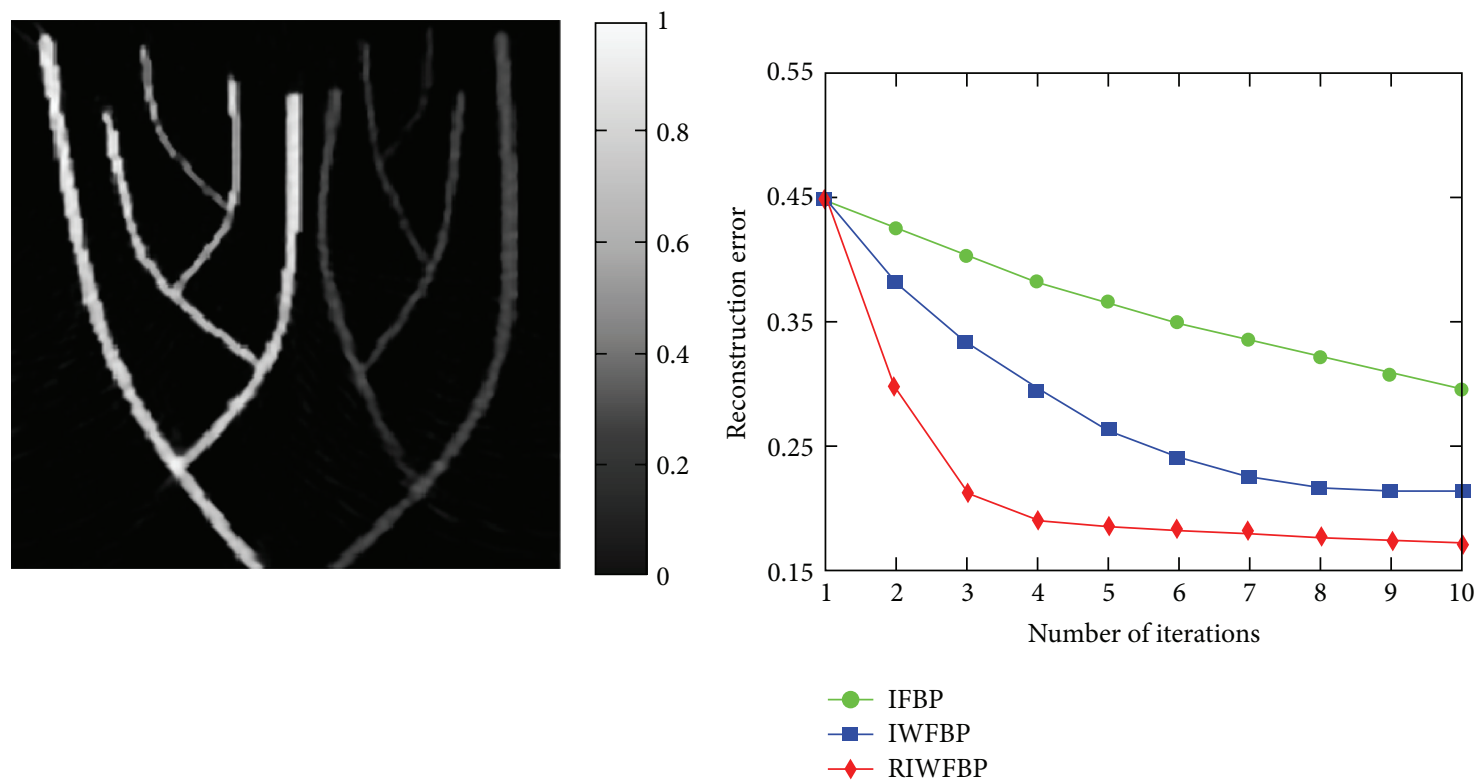

(c)
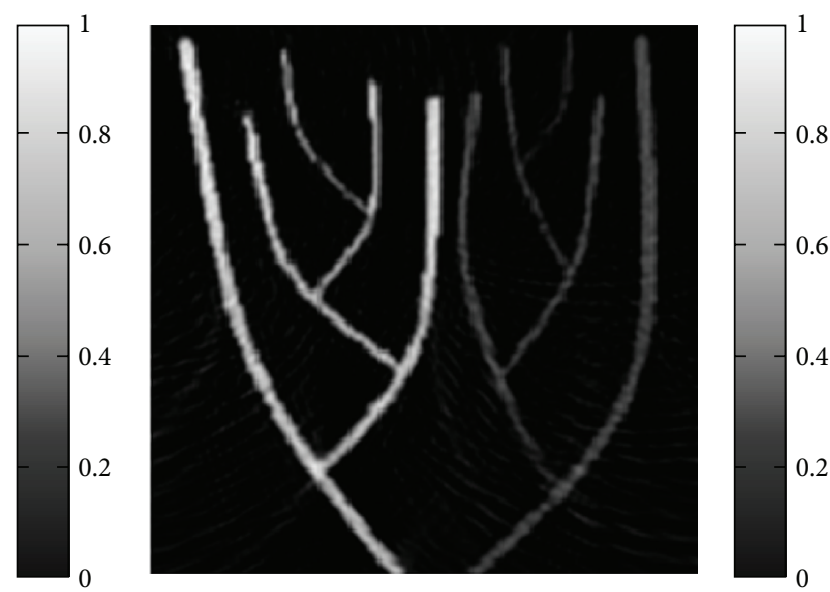

(b)

(d)

FIGURE 2: Photoacoustic imaging of the numerical phantom using 60 detections over $180^{\circ}$. (a) IFBP; (b) IWFBP; (c) RIWFBP. (d) Reconstruction normalized mean absolute error from $180^{\circ}$ under different number of iterations.

Finally, the last term in (9) has been added to the update step in (6), resulting in

$$
x^{m}=x^{m-1}+\alpha\left[R\left(y-\lambda_{m-1} P x^{m-1}\right)-\gamma K x^{m-1}\right] .
$$

The convergence of the iteration has been explained by Sunnegådh and Danielsson [22]. In this paper, the quality of the reconstructed image is measured via the normalized mean absolute error (NMAE), which is most sensitive to distortion artifacts defined as

$$
\delta_{i}=\frac{\left\|x_{i}-x\right\|_{2}}{\|x\|_{2}} .
$$

After a few iterations, artifacts are suppressed while the edge and detailed information is preserved well. When a desired minimum NMAE has been achieved the iterative process will stop, and then the results will be output.

\section{Results}

3.1. Reconstructions from Simulated Few-View Data. Computer simulations were conducted to demonstrate the effectiveness of the proposed method. The imaged source with a size of $256 \times 256$ pixels, as shown in Figure 1(a), was approximately located within a thin slab. The photoacoustic signals were calculated according to (1), and Gaussian noises were also added to simulated signals. In the experiments, all reconstruction methods were implemented in MATLAB (MathWorks, Natick, MA).

Images reconstructed with the IFBP method and the RIWFBP method from few-view data are displayed in Figure 1, respectively. There are many artifacts and blurs in Figures $1(\mathrm{~b})$ and $1(\mathrm{c})$. We hypothesize that the distortions present in Figures 1(b) and 1(e) come from the limited information available. This result is in agreement with the theoretical 


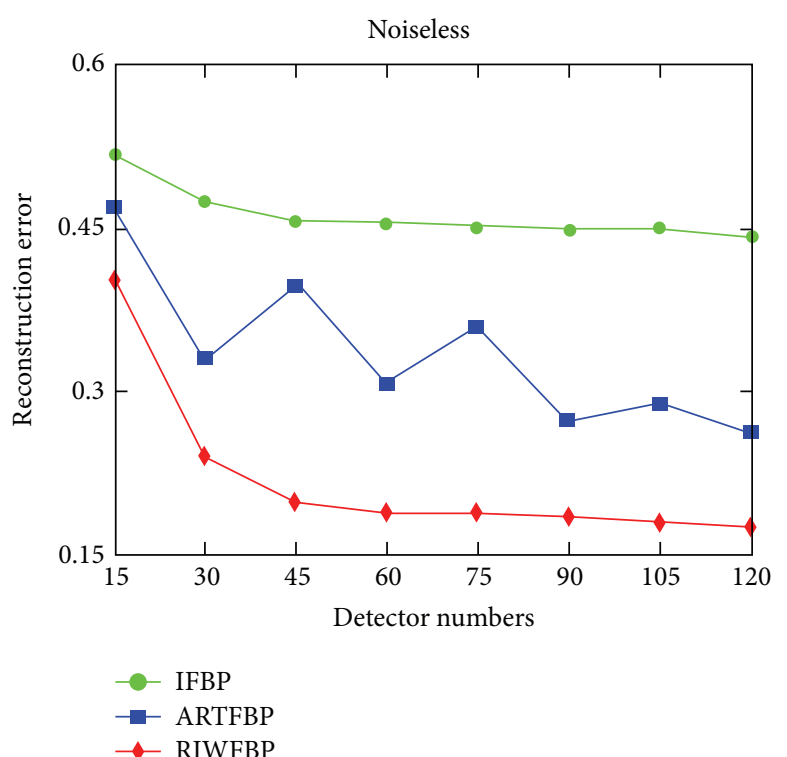

(a)

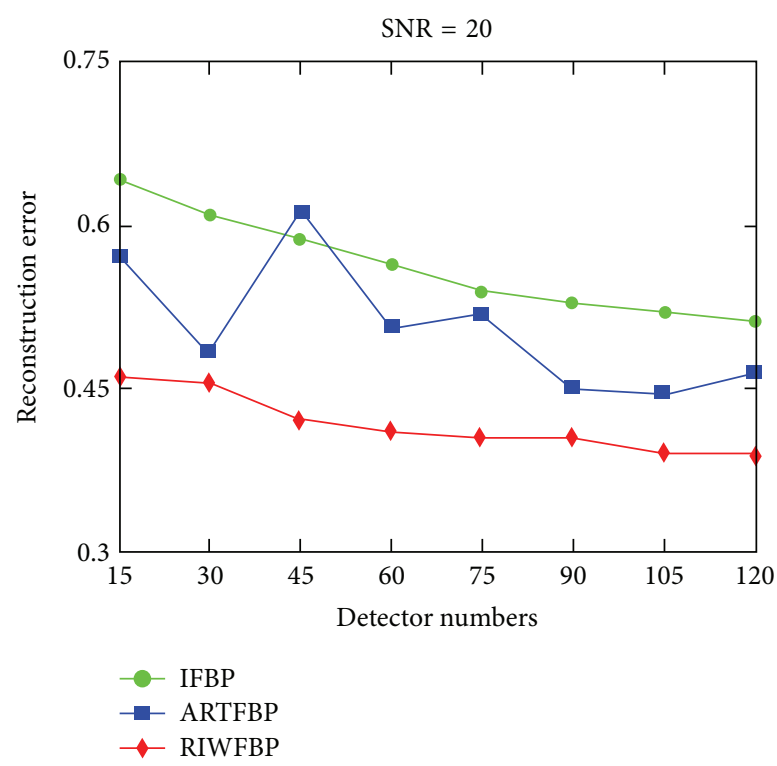

(c)

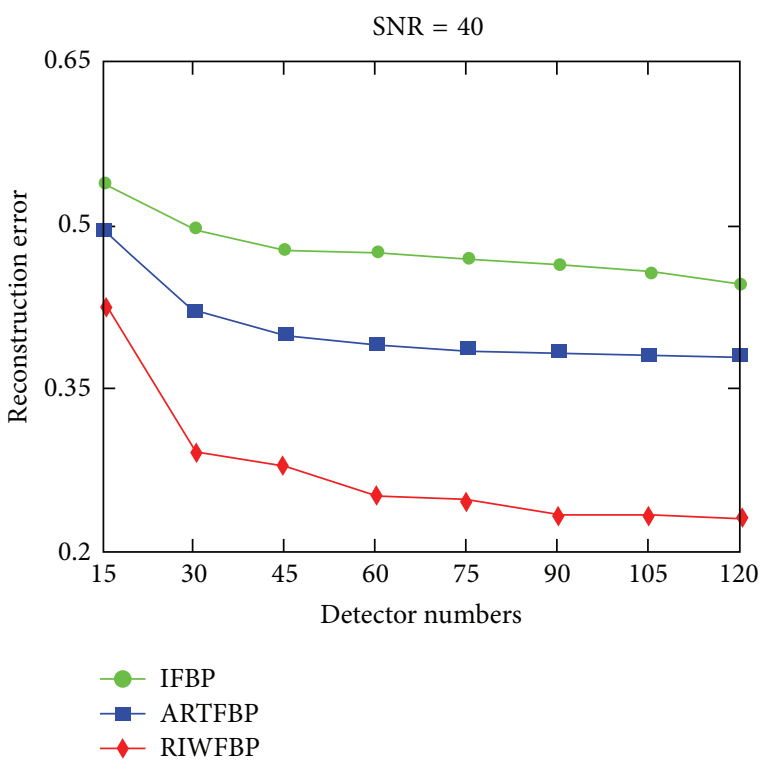

(b)

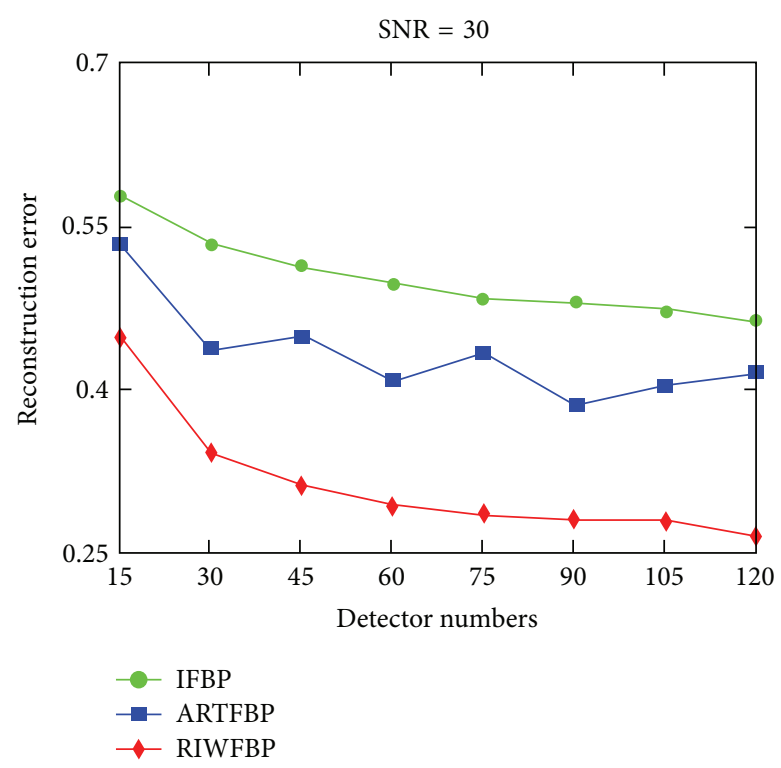

(d)

FIGURE 3: Reconstruction normalized mean absolute errors from $180^{\circ}$ with a different detector number. (a) Noiseless observation; noisy observation with (b) SNR = $40 \mathrm{~dB}$; (c) SNR = $30 \mathrm{~dB}$; (d) SNR = $20 \mathrm{~dB}$.

prediction in [8]. When comparing Figures $1(\mathrm{~d})$ and $1(\mathrm{f})$, it can be seen that the quality of the image reconstructed from few-view data is comparable with that from full-view. So we can conclude that with the incomplete data, the RIWFBP method can improve the quality of reconstruction with fewview data.

Figures 2(a)-2(c) show the reconstructions utilizing the IFBP method, the IWFBP method [26], and the RIWFBP method with 60 detections over $180^{\circ}$, respectively. It is obvious that the artifacts clearly seen in Figure 2(a) are suppressed to a large degree in Figure 2(c). By choosing an appropriate value for the regularization parameter $\gamma$, the artifact characteristics of the FBP method can be preserved.
The nonregularized IWFBP is clearly outperformed by the regularized.

Reconstruction errors between the original image and the reconstructed images were calculated and shown in Figure 2(d). We can see that the NMAE of the proposed method decrease especially when the iterative numbers are fewer. The reconstruction error for the RIWFBP falls to 0.18 in the first five iterations and continues to drop down to 0.17 after 10 iterations. In contrast to the IFBP method and the IWFBP method, the proposed method is expected to deliver a good result in less than five iterations.

To study the robustness of the RIWFBP method, Gaussian noises with SNR 40, 30, and 20 were added to the signals. 


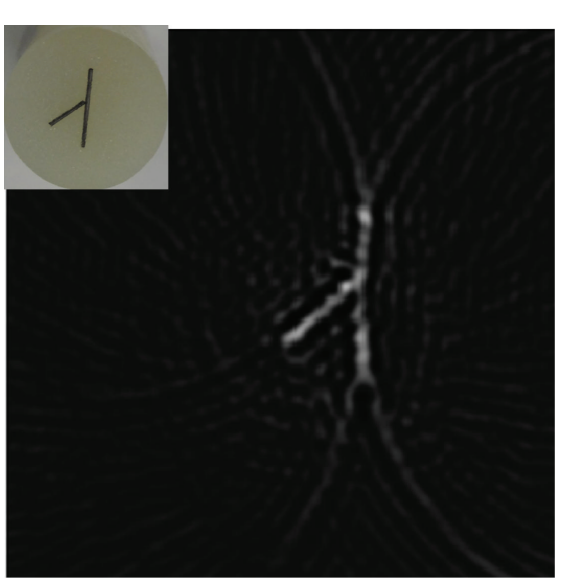

(a)
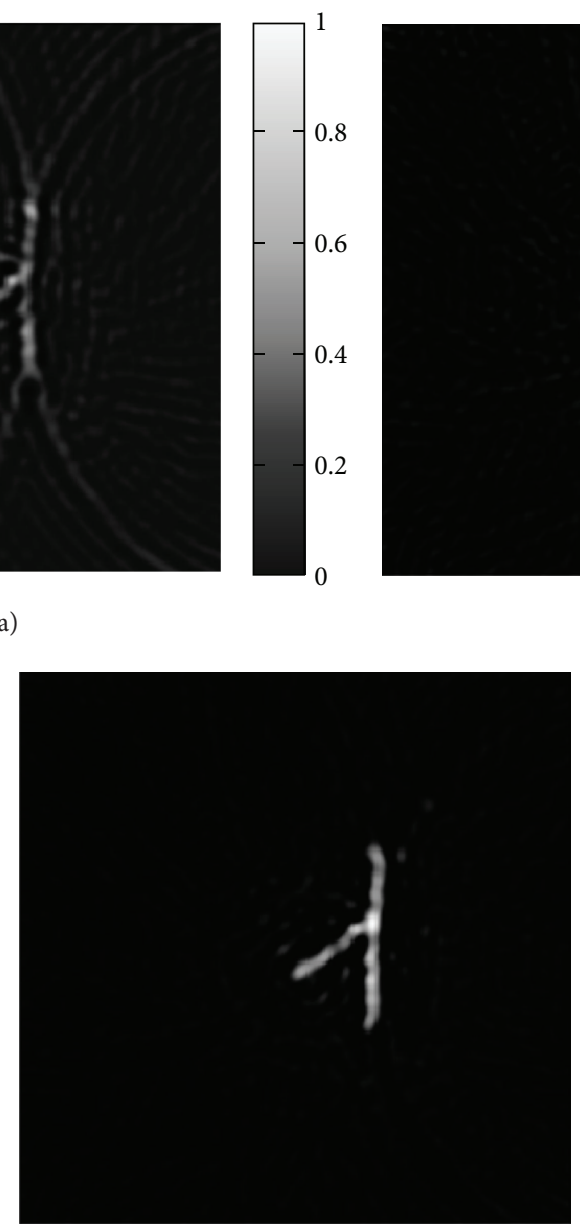

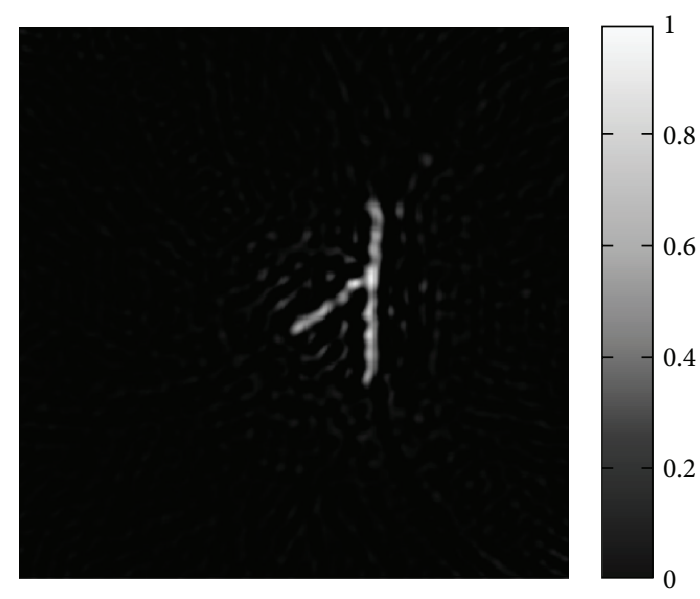

(b)

(c)

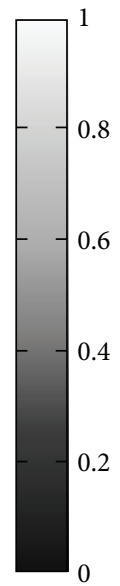

FIGURE 4: Reconstructed images based on few-view data. (a) 60 detectors over $180^{\circ}$, with FBP. The insert at the top-left corner is the photograph of the phantom. (b) 60 detectors over $180^{\circ}$, with IFBP. (c) 60 detectors over $180^{\circ}$, with RIWFBP.

Figure 3 shows the tendency chart of reconstruction errors between the phantom and the reconstruction obtained from the IFBP method, the ARTFBP method [27, 28], and the RIWFBP method. It can be demonstrate that the RIWFBP method has the minimum NMAE. The regularized iterative scheme is robust to inaccurate measurements and has greater improvement in calculation accuracy as predicted by the theory presented in [8]. The RIWFBP method can not only use the fewest measurements to get the best performance, but also maintain the best effects whether the signals are noisy or noiseless.

3.2. PAI Imaging of the Phantom. In the phantom experiment, the RIWFBP method was tested and evaluated. For comparative purposes, reconstruction results of the FBP method and the IFBP method are also presented. Two graphite rods with a diameter $r=0.5 \mathrm{~mm}$ and lengths of $5 \mathrm{~mm}$ and $10 \mathrm{~mm}$ were buried in a cylindrical phantom at a depth of $4 \mathrm{~mm}$. The phantom made of a mixture with $1 \%$ intralipid, $6 \%$ gelatin, and $93 \%$ water was used to simulate biological tissues. The inset in Figure 4(a) is the photograph of the phantom.
The sample was irradiated with pulses from a Q-switched Nd: YAG Laser. The pulse duration was $7.5 \mathrm{~ns}$ and the pulse repetition rate was $10 \mathrm{~Hz}$. A focused hydrophone (Precision Acoustics Ltd.) with frequency response of $5 \mathrm{MHz}$ was controlled by a high precision stepping motor to scan around the phantom in a circular manner for photoacoustic signal acquisition. The distance between the transducer and the rotation center was $45 \mathrm{~mm}$. The induced photoacoustic waves were captured at 60 positions over $180^{\circ}$. At each position 50 signals were averaged. In real experiments, we did not know the true image, so the iterative stop criteria,

$$
e_{i}=\frac{\left\|x_{i}-x_{i-1}\right\|_{2}}{\left\|x_{i}\right\|_{2}}<\varepsilon, \quad(i=1,2, \ldots, n),
$$

were taken.

Figures 4(a)-4(c) show the reconstructed photoacoustic image utilizing the FBP method, the IFBP method, and the RIWFBP method, respectively. As expected, the image reconstructed with the FBP method contains artifacts and distortions. The artifacts in Figure 4(b) are much smaller than 
those in Figure 4(a). There is almost no artifact in Figure 4(c) which is in good agreement with the phantom. The results of the phantom experiment demonstrate that the RIWFBP method works well with few-view photoacoustic data.

\section{Conclusions}

In this work, the RIWFBP method has been applied to reconstruct photoacoustic images with few-view data. From the experiments we conclude that during the first five iterations, the RIWFBP method efficiently suppresses strips artifacts produced by IWFBP over $180^{\circ}$. The NMAE calculation also denoted that the RIWFBP method has an advantage in accuracy compared with other test methods. With regularization, the proposed method reaches the final solution faster than without. It is therefore easier to decide when to terminate the iterative loop. The application of the RIWFBP method will significantly reduce the number of ultrasound transducers and scanning time needed for high quality photoacoustic image reconstruction. Therefore, it can be a promising candidate for resolving the few-view PAI problem.

\section{Competing Interests}

The authors declare that they have no competing interests.

\section{Acknowledgments}

This paper is supported by the National Natural Science Foundation of China under Grant nos. 31271065, 61300154, and 61402215, the Natural Science Foundation of Shandong Province under Grant nos. 2014ZRB019VC and 2014ZRB019E0, and the Doctoral Fund of Liaocheng University under Grant no. 318051304.

\section{References}

[1] L. V. Wang, "Prospects of photoacoustic tomography," Medical Physics, vol. 35, no. 12, pp. 5758-5767, 2008.

[2] L. V. Wang and S. Hu, "Photoacoustic tomography: in vivo imaging from organelles to organs," Science, vol. 335, no. 6075 , pp. 1458-1462, 2012.

[3] X. L. Deán-Ben, T. F. Fehm, and M. Gostic, "Volumetric handheld optoacoustic angiography as a tool for real-time screening of dense breast," Journal of Biophotonics, vol. 21, no. 2, pp. 194196, 2015.

[4] A. Garcia-Uribe, T. N. Erpelding, A. Krumholz et al., "DualModality photoacoustic and ultrasound imaging system for noninvasive sentinel lymph node detection in patients with breast cancer," Scientific Reports, vol. 5, Article ID 15748, 2015.

[5] M. Xu and L. V. Wang, "Universal back-projection algorithm for photoacoustic computed tomography," Physical Review E, vol. 71, no. 1, part 2, Article ID 016706, pp. 151-155, 2005.

[6] D. Yang, D. Xing, S. Yang, and L. Xiang, "Fast full-view photoacoustic imaging by combined scanning with a linear transducer array," Optics Express, vol. 15, no. 23, pp. 15566-15575, 2007.
[7] Z. Yuan and H. Jiang, "Quantitative photoacoustic tomography: recovery of optical absorption coefficient maps of heterogeneous media," Applied Physics Letters, vol. 88, no. 23, Article ID 231101, 2006.

[8] Y. Xu, L. V. Wang, G. Ambartsoumian, and P. Kuchment, "Reconstructions in limited-view thermoacoustic tomography," Medical Physics, vol. 31, no. 4, pp. 724-733, 2004.

[9] L. Yao and H. Jiang, "Photoacoustic image reconstruction from few-detector and limited-angle data," Biomedical Optics Express, vol. 2, no. 9, pp. 2649-2654, 2011.

[10] J. Meng, L. V. Wang, L. Ying, D. Liang, and L. Song, "Compressed-sensing photoacoustic computed tomography in vivo with partially known support," Optics Express, vol. 20, no. 15, pp. 16510-16523, 2012.

[11] J. Provost and F. Lesage, "The application of compressed sensing for photo-acoustic tomography," IEEE Transactions on Medical Imaging, vol. 28, no. 4, pp. 585-594, 2009.

[12] X. L. Deán-Ben, R. Ma, D. Razansky, and V. Ntziachristos, "Statistical approach for optoacoustic image reconstruction in the presence of strong acoustic heterogeneities," IEEE Transactions on Medical Imaging, vol. 30, no. 2, pp. 401-408, 2011.

[13] G. Paltauf, J. A. Viator, S. A. Prahl, and S. L. Jacques, "Iterative reconstruction algorithm for optoacoustic imaging," Journal of the Acoustical Society of America, vol. 112, no. 4, pp. 1536-1544, 2002.

[14] K. Wang, R. Su, A. A. Oraevsky, and M. A. Anastasio, "Investigation of iterative image reconstruction in three-dimensional optoacoustic tomography," Physics in Medicine \& Biology, vol. 57, no. 17, pp. 5399-5423, 2012.

[15] T. Saratoon, T. Tarvainen, T. B. Cox et al., "A gradient-based method for quantitative photoacoustic tomography using the radiative transfer equation," Inverse Problems, vol. 29, no. 7, pp. 75006-75024, 2013.

[16] C. Huang, K. Wang, L. Nie, L. V. Wang, and M. A. Anastasio, "Full-wave iterative image reconstruction in photoacoustic tomography with acoustically inhomogeneous media," IEEE Transactions on Medical Imaging, vol. 32, no. 6, pp. 1097-1110, 2013.

[17] K. Wang, C. Huang, Y.-J. Kao, C.-Y. Chou, A. A. Oraevsky, and M. A. Anastasio, "Accelerating image reconstruction in threedimensional optoacoustic tomography on graphics processing units," Medical Physics, vol. 40, no. 2, Article ID 023301, 2013.

[18] L. Ding, X. L. Deán-Ben, and D. Razansky, "Real-time modelbased inversion in cross-sectional optoacoustic tomography," IEEE Transactions on Medical Imaging, vol. 35, no. 8, pp. 18831891, 2016.

[19] J. Zhang, M. A. Anastasio, X. Pan, and L. V. Wang, "Weighted expectation maximization reconstruction algorithms for thermoacoustic tomography," IEEE Transactions on Medical Imaging, vol. 24, no. 6, pp. 817-820, 2005.

[20] X. L. Deán-Ben, R. Ma, A. Rosenthal, V. Ntziachristos, and D. Razansky, "Weighted model-based optoacoustic reconstruction in acoustic scattering media," Physics in Medicine \& Biology, vol. 58, no. 16, pp. 5555-5566, 2013.

[21] H. Huang, G. Bustamante, R. Peterson, and J. Y. Ye, "An adaptive filtered back-projection for photoacoustic image reconstruction," Medical Physics, vol. 42, no. 5, pp. 2169-2178, 2015.

[22] J. Sunnegårdh and P.-E. Danielsson, "Regularized iterative weighted filtered backprojection for helical cone-beam CT," Medical Physics, vol. 35, no. 9, pp. 4173-4185, 2008. 
[23] M. Magnusson, P.-E. Danielsson, and J. Sunnegårdh, "Handling of long objects in iterative improvement of nonexact reconstruction in helical cone-beam CT,' IEEE Transactions on Medical Imaging, vol. 25, no. 7, pp. 935-940, 2006.

[24] D. Wu, C. Tao, X.-J. Liu, and X.-D. Wang, "Influence of limitedview scanning on depth imaging of photoacoustic tomography," Chinese Physics B, vol. 21, no. 1, Article ID 014301, 2012.

[25] X. Wang, Y. Xu, M. S. Xu, S. Yokoo, E. S. Fry, and L. V. Wang, "Photoacoustic tomography of biological tissues with high cross-section resolution: reconstruction and experiment," Medical Physics, vol. 29, no. 12, pp. 2799-2805, 2002.

[26] X. Liu, D. Peng, X. Ma et al., "Limited-view photoacoustic imaging based on an iterative adaptive weighted filtered backprojection approach," Applied Optics, vol. 52, no. 15, pp. 34773483, 2013.

[27] L.-Z. Xiang, D. Xing, H.-M. Gu, D.-W. Yang, S.-H. Yang, and L.-M. Zeng, "Photoacoustic imaging of blood vessels based on modified simultaneous iterative reconstruction technique," Acta Physica Sinica, vol. 56, no. 7, pp. 3911-3916, 2007.

[28] D. Yang, "Limited-view scanning photoacoustic imaging based on algebraic reconstruction techniques," Acta Optica Sinica, vol. 6047, pp. 60470I-60470I-8, 2005. 


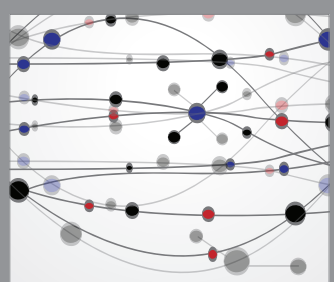

The Scientific World Journal
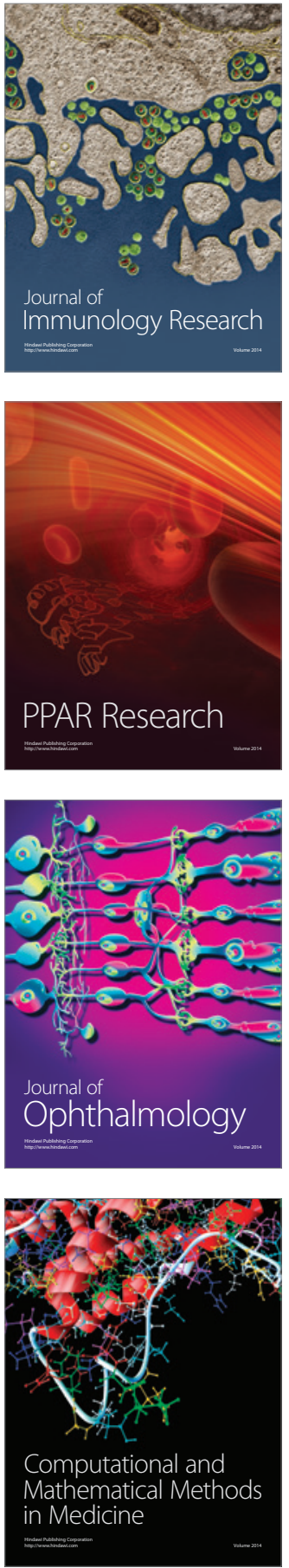

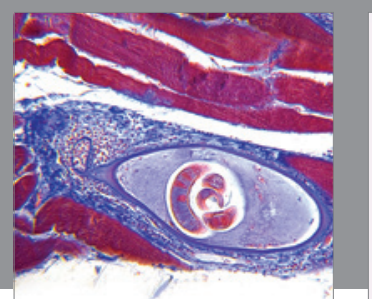

Gastroenterology Research and Practice

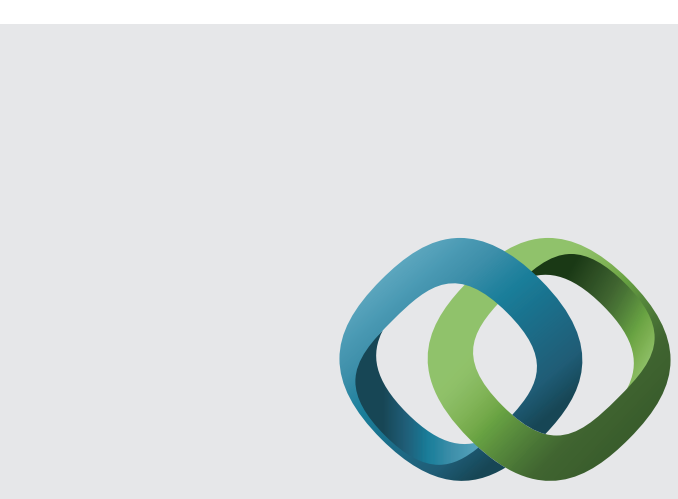

\section{Hindawi}

Submit your manuscripts at

http://www.hindawi.com
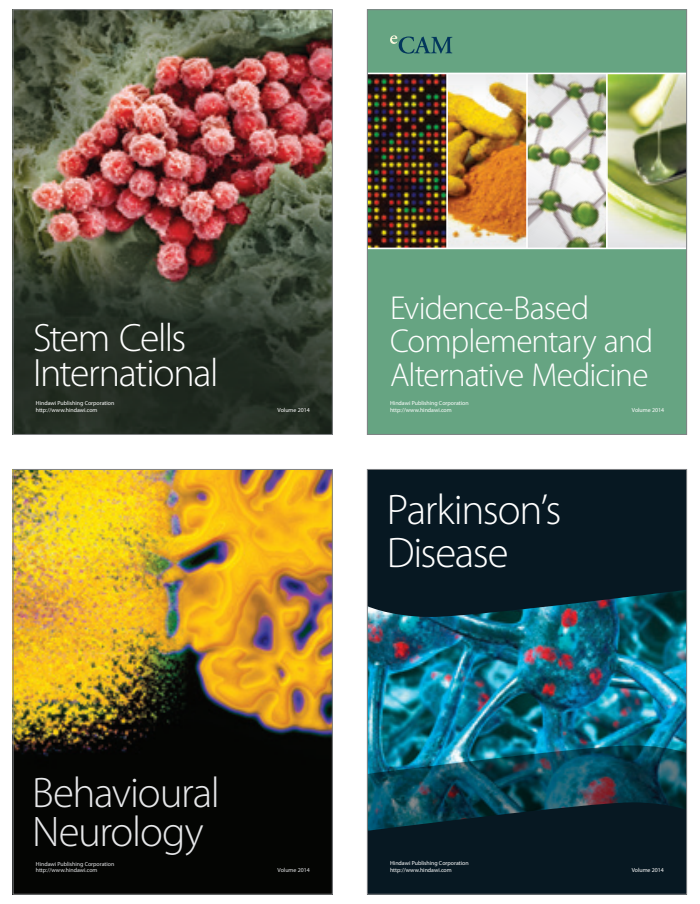
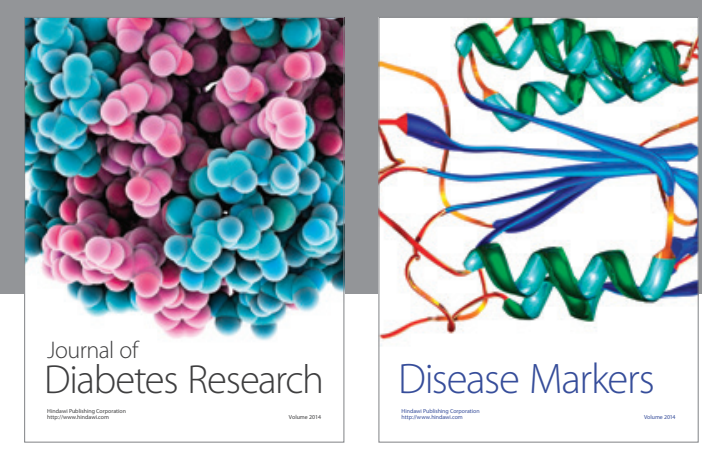

Disease Markers
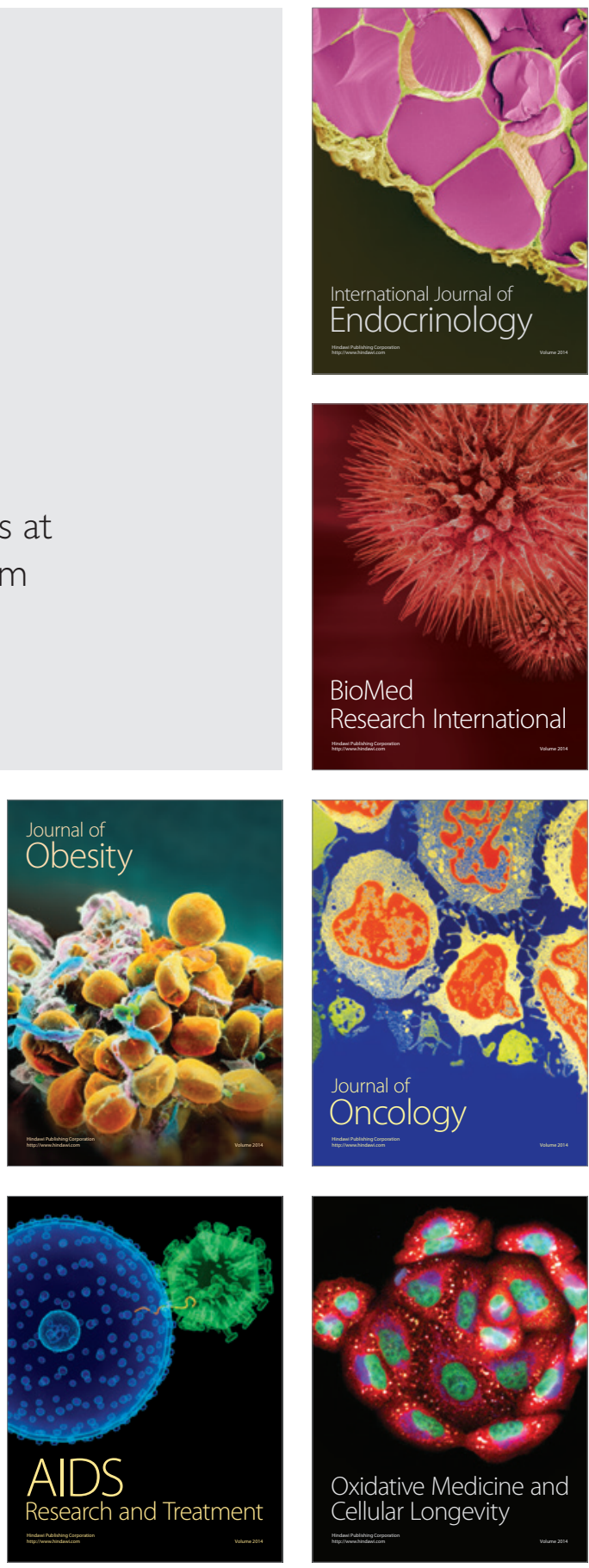